\title{
Commentary: Under pressure: The role of inflammation in aortic aneurysm pathophysiology
}

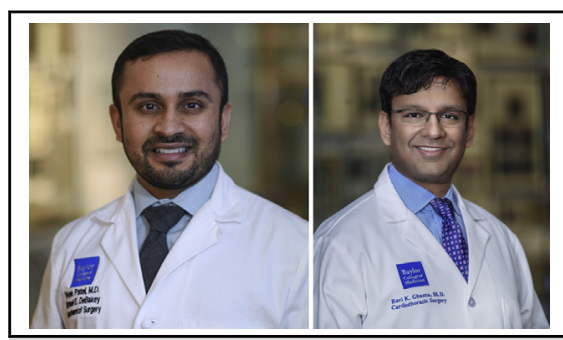

Vivek Patel, MD (left), and Ravi K. Ghanta, MD (right)

Central Message

The pathophysiology of aortic aneurysms is influenced by multiple processes including hemodynamic stress, aortic injury, chronic inflammation, genetic abnormalities, and individual comorbidities.

See Article page 1434 .
Although the consequences of aortic pathology are often fatal, the pathophysiology of aortic aneurysms and dissection remains poorly understood. The prevailing theory of cystic medial necrosis, a degenerative process thought to weaken the aortic wall, was first described by Gsell in 1928 and Erdhein in 1930. ${ }^{1}$ Recent studies, including the study presented by Leone and colleagues ${ }^{2}$ in this issue of the Journal, have uncovered that inflammation may actually play a greater role than previously described. In fact, degeneration and inflammation of the aortic wall may not be mutually exclusive processes but rather phases along the continuum of aortic pathology.

Building on their previous work, Leone and colleagues characterized the histopathology of 255 patients undergoing surgery for thoracic aortic aneurysms, determining that atherosclerosis $(18.8 \%)$ and aortitis $(13.7 \%)$ accounted for a significant portion of the samples. ${ }^{2,3}$ In total, $25 \%$ of patients had a mixed (degenerative-atherosclerosis, degenerative-aortitis or atherosclerosis-aortitis) profile. In addition, the prevailing histopathologic profiles correlated to the patients' comorbidities. Patients with bicuspid valves were more likely to have a degenerative profile, whereas older patients with diabetes, a history of tobacco abuse, and coronary artery disease had a predominantly atherosclerotic profile. Patients in the aortitis cohort had larger aneurysm diameters, were primarily female, and had greater body mass index.

Leone and colleague's study exemplifies the complexity of aortic pathophysiology, which we now understand is a combination of hemodynamic stress, aortic injury, chronic inflammation, genetic abnormalities, and individual comorbidities. ${ }^{1,4,5}$ Emerging studies now indicate that degeneration of the aortic wall simply represents one phase of the natural history of aortic disease and that inflammation may be the initial insult. Wu and colleagues ${ }^{6}$ showed that the NLRP3-caspase-1 inflammasome cascade-mediated degradation of smooth muscle cells contributes to aortic biomechanical dysfunction and aneurysm development. In addition, others have implicated transforming growth factor-beta dysregulation, natural killer cells, and autoimmunity following viral or bacterial infections as other possible explanations for aortic wall degradation. ${ }^{4,5}$ Pharmacologic treatments that inhibit these pathways, such as glyburide-mediated inhibition of the inflammasome cascade, may protect against aortic aneurysms. ${ }^{6}$

Leone and colleagues' work further validates the role of inflammation in aortic pathophysiology, but several considerations come to light. First, the histopathologic survey presented here represents a snapshot along the natural history of aortic disease, limiting our ability to understand the temporal association between these events. A chronological study evaluating specimens along the timeline of aortic disease is, of course, only possible in animal models. Second, the authors grouped samples from patients with aneurysms, dissection, intramural hematomas, and aortic ulcers, which may further limit our ability to discern a pattern between these processes. Third, it is difficult to draw conclusions from the small cohort $(n=5)$ of patients with Marfan's syndrome presented here. Connective tissue disorders leading to aortic disease may be best to be studied separately.

Taken together, this study underscores the role of inflammation in aortic pathophysiology. The authors provide us with a better understanding of correlation between histopathology and clinical risk factors, which may help identify 
patients at greater risk. A deeper understanding of the complex relationship between inflammation and degeneration at the molecular level is expected to help with the development of targeted therapies. ${ }^{4-6}$

\section{References}

1. Wu D, Shen YH, Russell L, Coselli JS, LeMaire SD. Molecular mechanisms of thoracic aortic dissection. J Surg Res. 2013;184:907-24.

2. Leone O, Corsini A, Pacini D, Corti B, Laus V, Foa A, et al. The complex interplay between atherosclerosis, inflammation and degeneration in ascending thoracic aortic aneurysms. J Thorac Cardiovasc Surg. 2020;160:1434-43.e6.
3. Leone O, Pacini D, Foa A, Corsini A, Agostini V, Corti B, et al. Redefining the histopathologic profile of acute aortic syndromes: clinical and prognostic implications. J Thorac Cardiovasc Surg. 2018;156:1776-85.

4. Kim HW, Stansfield BK. Genetic and epigenetic regulation of aortic aneurysms BioMed Res Int. 2017;2017:7268521.

5. Kuivaniemi H, Ryer EJ, Elmore JR, Tromp G. Understanding the pathogenesis of abdominal aortic aneurysms. Expert Rev Cardiovasc Ther. 2015;13:975-87.

6. Wu D, Ren P, Zheng Y, Zhang L, Xu G, Xie W, et al. NLRP3 (nucleotide oligomerization domain-like receptor family, pyrin domain containing 3)-caspase-1 inflammasome degrades contractile proteins: implications for aortic biomechanical dysfunction and aneurysm and dissection formation. Arterioscler Thromb Vasc Biol. 2017;37:694-706. 